\title{
Broadband absorption engineering to enhance light absorption in monolayer $\mathrm{MoS}_{2}$
}

\author{
Shah Mohammad Bahauddin ${ }^{\dagger} \| \perp$, Hossein Robatjazi $i^{\dagger} \| \perp$ and Isabell Thomann $* \dot{\dagger}, \dot{+}, \xi, \|, \perp$ \\ ${ }^{\dagger}$ Department of Electrical and Computer Engineering, $\ddagger$ Department of Materials Science and \\ NanoEngineering, §Department of Chemistry, "Laboratory for Nanophotonics, and ${ }^{\perp}$ Rice \\ Quantum Institute, Rice University, 6100 Main Street, Houston, Texas 77005, United States.
}

Corresponding Author

* Email: isabell.thomann@,rice.edu 


\begin{abstract}
Here we take a first step towards tackling the challenge of incomplete optical absorption in monolayers of transition metal dichalcogenides for conversion of photon energy, including solar, into other forms of energy. We present a monolayer $\mathrm{MoS}_{2}$-based photoelectrode architecture that exploits nanophotonic light management strategies to enhance absorption within the monolayer of $\mathrm{MoS}_{2}$, while simultaneously integrating an efficient charge carrier separation mechanism facilitated by a $\mathrm{MoS}_{2} / \mathrm{NiO}_{\mathrm{x}}$ heterojunction. Specifically, we demonstrate two extremely thin photoelectrode architectures for solar-fuel generation: (i) A planar optical cavity architecture $\mathrm{MoS}_{2} / \mathrm{NiO}_{\mathrm{x}} / \mathrm{Al}$ that improves optical impedance matching and (ii) an architecture employing plasmonic silver nanoparticles (Ag NPs) $\mathrm{MoS}_{2} / \mathrm{Ag} \mathrm{NPs} / \mathrm{NiO}_{\mathrm{x}} / \mathrm{Al}$ that further improves light absorption within the monolayer. We used a combination of numerical simulations, analytical models and experimental optical characterizations to gain insights in the contributions of optical impedance matching versus plasmonic near-field enhancement effects in our plasmonic photoelectrode structures. By performing three-dimensional electromagnetic simulations, we predict structures that can absorb $37 \%$ of the incident light integrated from 400 to $700 \mathrm{~nm}$ within a monolayer of $\mathrm{MoS}_{2}$, a 5.9x enhanced absorption compared to that of $\mathrm{MoS}_{2}$ on a sapphire $\left(\mathrm{Al}_{2} \mathrm{O}_{3}\right)$ substrate. Experimentally, a 3.9x absorption enhancement is observed in the total structure compared to that of $\mathrm{MoS}_{2} / \mathrm{Al}_{2} \mathrm{O}_{3}$, and photoluminescence measurements suggest this enhancement largely arises from absorption enhancements within the $\mathrm{MoS}_{2}$ layer alone. The results of these measurements also confirm that our $\mathrm{MoS}_{2} / \mathrm{NiO}_{\mathrm{x}} / \mathrm{Al}$ structures do indeed facilitate efficient charge separation, as required for a photoelectrode. To rapidly explore the parameter space of plasmonic photoelectrode architectures we also developed an analytical model based on an effective medium model that is in excellent agreement with results from numerical FDTD simulations.
\end{abstract}


KEYWORDS: Monolayer molybdenum disulfide $\left(\mathrm{MoS}_{2}\right)$, two-dimensional (2D) materials, plasmonics, absorption engineering, photoelectrode architecture, photocatalysis. 
Monolayer $\mathrm{MoS}_{2}$ is a promising material for several classes of optoelectronic devices ${ }^{1-9}$ and photocatalytic applications ${ }^{10-12}$. In contrast to multilayer $\mathrm{MoS}_{2}$, monolayer $\mathrm{MoS}_{2}$ has a direct band gap around $1.8 \mathrm{eV}(690 \mathrm{~nm})$. This band gap is ideal for a photoelectrode for solar water splitting based on a single semiconductor ${ }^{11}$ and enables broadband light absorption in the visible and ultraviolet regions. Furthermore, the bandgap of monolayer $\mathrm{MoS}_{2}$ is ideal for tandem cells which achieve highest power conversion efficiencies if two materials with band gaps of $1.1 \mathrm{eV}$ and 1.8 $\mathrm{eV}$ are combined ${ }^{5,11,13-15}$. The electronic structure and the conduction and valence band edge positions of monolayer $\mathrm{MoS}_{2}$, in contrast to multilayer $\mathrm{MoS}_{2}$, are also near-ideal for driving the water splitting reactions ${ }^{11,16,17}$. Furthermore, $\mathrm{MoS}_{2}$ is currently extensively explored as a nonprecious hydrogen evolution reaction catalyst ${ }^{10,11,18-21}$ with a performance potentially rivaling that of expensive platinum. Notwithstanding the unique properties that monolayer transition metal dichalcogenides (TMDC) such as molybdenum disulfide $\left(\mathrm{MoS}_{2}\right)$ have to offer, the low light absorption in monolayers limits their use in photocatalytic applications and energy-efficient optoelectronic devices ${ }^{22,23}$. Therefore, intensive research efforts worldwide are directed at achieving efficient and application-tailored light absorption within two-dimensional monolayer $\operatorname{architectures}^{24-27}$.

Monolayer $\mathrm{MoS}_{2}$ absorbs less than $8 \%{ }^{13}$ of the incident light in the 400 to $700 \mathrm{~nm}$ range when supported on a planar silica substrate. A recent numerical study predicted the possibility of broadband absorption of up to $33 \%$ in monolayer $\mathrm{MoS}_{2}$ that was sandwiched within a sophisticated chirped distributed Bragg cavity design, intended for a photodetector application ${ }^{24}$. Another approach to enhance light absorption within nanoscale regions is the utilization of plasmonic nanoantennas ${ }^{28,29,30}$. A first demonstration of plasmon-enhanced light absorption within monolayer $\mathrm{MoS}_{2}$ was reported by Britnell et al. ${ }^{31}$ who spattered gold nanoparticles on top of $\mathrm{MoS}_{2}$ 
and observed a 10-fold increase in the photocurrent at $633 \mathrm{~nm}$. Sobhani et al. ${ }^{32}$ employed silicacore gold-shell nanoparticles with a surface coverage of less than $1 \%$ and demonstrated a 3 -fold increase in the photocurrent and a 2 -fold increase in the photoluminescence at the excitonic transitions of $630 \mathrm{~nm}$ and $680 \mathrm{~nm}$ of $\mathrm{MoS}_{2}$ near the band-edge. While plasmonic gold nanostructures can enhance light absorption and photoluminescence near the band gap of $\mathrm{MoS}_{2}$, they are not suitable for broadband absorption enhancements required for solar energy conversion devices, since the interband transitions in gold tend to suppress light absorption at short wavelengths.

In this article, we explore novel photoelectrode architectures based on single-layer molybdenum disulfide $\left(\mathrm{MoS}_{2}\right)$ that employ broadband absorption engineering strategies from 400 to $700 \mathrm{~nm}$, while simultaneously incorporating an efficient charge carrier separation mechanism. First, we investigated a planar optical cavity $\mathrm{MoS}_{2} / \mathrm{NiO}_{\mathrm{x}} / \mathrm{Al}$ that improves optical impedance matching. Optical impedance matching is achieved when reflection from the architecture can be completely suppressed, i.e. the amplitude and phase of the first reflection coefficient are perfectly canceled by all the other reflections. Secondly, to increase light absorption within the monolayer even further, we designed an architecture employing plasmonic silver nanoparticles, $\mathrm{MoS}_{2} / \mathrm{Ag} \mathrm{NPs} / \mathrm{NiO}_{\mathrm{x}} / \mathrm{Al}$. The plasmonic structure can absorb up to $37 \%$ of the incident light within the monolayer of $\mathrm{MoS}_{2}$ integrated from 400 to $700 \mathrm{~nm}$ based on finite difference time domain (FDTD) simulations. Photoluminescence measurements from $\mathrm{MoS}_{2}$ are correlated with absorption measurements, and show that absorption indeed occurs within the monolayer rather than elsewhere in the photoelectrode architecture. These measurements also confirm that $\mathrm{MoS}_{2} / \mathrm{NiO}_{\mathrm{x}} / \mathrm{Al}$ structures facilitate efficient charge separation, as required for a photoelectrode. Since three-dimensional electromagnetic simulations can be time-consuming, we explored whether analytical models can 
be used to predict the tuning parameters for enhanced light absorption. We found that absorption within our full plasmonic photoelectrode architecture can be described in very good agreement with full-field electromagnetic simulations.

\section{Results and Discussion}

Figure 1a shows the measured and simulated absorbed fraction as a function of wavelength for $\mathrm{MoS}_{2} / 40 \mathrm{~nm} \mathrm{NiO} / \mathrm{Al}$ where a $40 \mathrm{~nm} \mathrm{NiO}$ spacer thickness provides the best optical impedance matching as predicted by three-dimensional FDTD simulations. As a control, we also fabricated $\mathrm{MoS}_{2} / 45 \mathrm{~nm} \mathrm{Al} \mathrm{O}_{3} / \mathrm{Al}$ (Figure 1b), an optimally impedance-matched structure that does not support charge carrier separation since $\mathrm{Al}_{2} \mathrm{O}_{3}$ is an insulator, but forms a near-identical photonic cavity since the dielectric constants of $\mathrm{Al}_{2} \mathrm{O}_{3}$ are very close to those of $\mathrm{NiO}_{\mathrm{x}}$. Absorption enhancements were referenced to planar $\mathrm{MoS}_{2}$ on $\mathrm{SiO}_{2}\left(\mathrm{Al}_{2} \mathrm{O}_{3}\right)$ substrates, respectively. Figures $1 \mathrm{a}$ and $1 \mathrm{~b}$ show the experimental and simulated absorption spectra in these structures. From simulations we find that approximately $74 \%$ of the incident light is absorbed within $\mathrm{MoS}_{2}(88 \%$ in total structure) at $425 \mathrm{~nm}$ (Supporting Information S1), corresponding to a six times stronger absorption at $425 \mathrm{~nm}$ compared to that of $\mathrm{MoS}_{2}$ on a glass $\left(\mathrm{SiO}_{2}\right)$ substrate. Experimentally, the absorbed fraction rises to $69 \%$ at $450 \mathrm{~nm}$ for $\mathrm{MoS}_{2} / \mathrm{NiO}_{\mathrm{x}} / \mathrm{Al}$ compared to $12 \%$ for $\mathrm{MoS}_{2}$ on $\mathrm{SiO}_{2}$ (Figure 1). The good agreement between simulations and experimental results in these planar $\mathrm{MoS}_{2}$ architectures shows that better optically impedance matched architectures can strongly enhance light absorption in monolayer $\mathrm{MoS}_{2}$ - a first step towards energy-efficient optoelectronic and photocatalytic devices that would benefit from the unique electronic and catalytic properties of monolayer $\mathrm{MoS}_{2}$. 
To get a more direct experimental assessment of the increased absorbed fraction within the monolayer $\mathrm{MoS}_{2}$ alone, we carried out a set of absorption and photoluminescence measurements in the same sample. We note that these were performed on a slightly less optimally impedance matched structure in which the dielectric spacer layer was too thin $\left(40 \mathrm{~nm} \mathrm{Al}_{2} \mathrm{O}_{3}\right)$ to achieve optimum absorption in the monolayer, but the conclusions are robust against small variations in device parameters.

To get a more direct experimental assessment of the increased absorbed fraction within the monolayer $\mathrm{MoS}_{2}$ alone we performed localized photoluminescence (PL) measurements of $\mathrm{MoS}_{2}$ on three substrates: (i) $\mathrm{Al}_{2} \mathrm{O}_{3}$ (sapphire), (ii) $40 \mathrm{~nm} \mathrm{Al}_{2} \mathrm{O}_{3} / \mathrm{Al}$, and (iii) $40 \mathrm{~nm} \mathrm{NiO} / \mathrm{Al}$. Figure 2 shows the variation of the PL signal strength of monolayer $\mathrm{MoS}_{2}$ acquired on sample (i), a triangular flake of single-layer $\mathrm{MoS}_{2}$ on $\mathrm{Al}_{2} \mathrm{O}_{3}$ and at approximately the same location after transferring the $\mathrm{MoS}_{2}$ to $40 \mathrm{~nm} \mathrm{Al} \mathrm{O}_{3} / \mathrm{Al}$ (sample (ii)). We also show the PL signal obtained from sample (iii), $\mathrm{MoS}_{2}$ on $40 \mathrm{~nm} \mathrm{NiO} / \mathrm{Al}$ exfoliated from $\mathrm{SiO}_{2}$ growth substrate. The presence of the strong A / A- exciton peak around $670 \mathrm{~nm}$ is a clear indication of the direct gap transition at the $\mathrm{K}$ point of the Brillouin zone in monolayer $\mathrm{MoS}_{2}$ and becomes much weaker for multilayer $\mathrm{MoS}_{2}{ }^{33-}$ 35. For the $\mathrm{MoS}_{2} / 40 \mathrm{~nm} \mathrm{Al} \mathrm{O}_{3} / \mathrm{Al}$ architecture, we find that the PL signal strength increases $3.2 \mathrm{x}$ compared to $\mathrm{MoS}_{2} / \mathrm{Al}_{2} \mathrm{O}_{3}$ (Figure 2). The close correspondence of this PL enhancement, arising from $\mathrm{MoS}_{2}$ alone, to the above-mentioned measured $3.9 \mathrm{x}$ absorption enhancement in the total structure (Supporting Information S2) demonstrates that the improved light absorption occurred largely within the monolayer of $\mathrm{MoS}_{2}$. We note that no strong additional enhancement due to emission outcoupling ${ }^{36}$ is anticipated because the electric field has no antinode in the $\mathrm{MoS}_{2}$ at the photoluminescence wavelength of $670 \mathrm{~nm}$ (Figure 5a). This attribution is furthermore supported by numerical simulations that predict an increase of absorption within the monolayer from $6.3 \%$ 
for $\mathrm{MoS}_{2} / \mathrm{Al}_{2} \mathrm{O}_{3}$ to $25.1 \%$ for $\mathrm{MoS}_{2} / 40 \mathrm{~nm} \mathrm{Al} \mathrm{O}_{3} / \mathrm{Al}$ (i.e. an enhancement of $4 \mathrm{x}$ ) (Supporting Information S3). A similar improvement in light absorption within the monolayer of $\mathrm{MoS}_{2}$ is expected for $\mathrm{MoS}_{2} / 40 \mathrm{~nm} \mathrm{NiO} / \mathrm{Al}$ since the dielectric constants of $\mathrm{NiO}_{\mathrm{x}}$ are close to those of $\mathrm{Al}_{2} \mathrm{O}_{3}$.

When instead PL is measured from $\mathrm{MoS}_{2}$ on the $\mathrm{NiO}_{\mathrm{x}}$ spacer/Al layer, the PL strength of monolayer $\mathrm{MoS}_{2}$ is reduced by $2.5 \mathrm{x}$ (Figure 2) or even more (Supporting Information S4) compared to that of $\mathrm{MoS}_{2} / \mathrm{Al}_{2} \mathrm{O}_{3}$ (sapphire substrate), which is surprising given the apparent integrated enhancement of the absorption of three times in the 400 to $700 \mathrm{~nm}$ region. Since the photonic cavity is the same (the refractive index for both wide band gap dielectric spacer layers $\mathrm{NiO}_{x}$ and $\mathrm{Al}_{2} \mathrm{O}_{3}$ is approximately $\mathrm{n}=1.8$ ) we can exclude any optics-based explanation for the reduced photoluminescence, and instead attribute the reduction of the PL strength to hole scavenging by the underlying $\mathrm{NiO}_{\mathrm{x}}$ substrate. The Raman signal strengths from $\mathrm{MoS}_{2}$ support the conclusions drawn from these PL measurements (Supporting Information S5): We observed a 17x enhancement in the Raman signal (area under the peaks at $387 \mathrm{~cm}^{-1}$ and $405 \mathrm{~cm}^{-1}$ ) for monolayer $\mathrm{MoS}_{2}$ on top of $\mathrm{Al}_{2} \mathrm{O}_{3} / \mathrm{Al}$ compared to an $\mathrm{Al}_{2} \mathrm{O}_{3}$ substrate when excited by a $514.5 \mathrm{~nm}$ laser. We attribute the strong Raman signal to the increased absorption in $\mathrm{MoS}_{2}$ and an improved outcoupling from our structure at $514 \mathrm{~nm}^{36}$ (Figure 5). The Raman signal was about 4x stronger for $\mathrm{MoS}_{2} / \mathrm{NiO}_{\mathrm{x}} / \mathrm{Al}$ than that for $\mathrm{MoS}_{2} / \mathrm{SiO}_{2}$ (Supporting Information S5), and we assume here that hole transfer from $\mathrm{MoS}_{2}$ to -type $\mathrm{NiO}_{\mathrm{x}}$ upon photoexcitation of $\mathrm{MoS}_{2}$ populates the electronic ground state and reduces outcoupling.

To explain this hole scavenging effect, we draw an energy band schematic for the $\mathrm{MoS}_{2} / \mathrm{NiO}_{\mathrm{x}}$ and $\mathrm{MoS}_{2} / \mathrm{Al}_{2} \mathrm{O}_{3}$ interface (Figure 3). The Fermi levels of a single layer of $\mathrm{MoS}_{2}$ and $\mathrm{NiO}_{\mathrm{x}}$ were previously reported at $4.7 \mathrm{eV}^{37-39}$ and $5.0 \mathrm{eV}^{40-42}$ resulting in a $0.3 \mathrm{eV}$ energy level offset (Figure 
3a), which causes an electrostatic field at the heterojunction interface that can facilitate charge carrier separation, and represents a promising alternative to recently investigated type II twodimensional (2D) heterojunctions ${ }^{43-45}$. Upon illumination, the photogenerated holes of $\mathrm{MoS}_{2} \mathrm{drift}$ to the energetically more favorable valence band of $\mathrm{NiO}_{x}$, leaving the electron behind; this spatial separation of charge carriers leads to the observed PL quenching in the $\mathrm{MoS}_{2} / \mathrm{NiO}_{\mathrm{x}} / \mathrm{Al}$ structure (Figure 2). We performed Mott-Schottky (M-S) measurements ${ }^{46-50}$ in a three-electrode electrochemical cell setup to verify the p-type properties ${ }^{40,42,50,51}$ of our $40 \mathrm{~nm}$ thick $\mathrm{NiO}_{\mathrm{x}}$ thin films on an $\mathrm{Al}$ back electrode. When instead $\mathrm{Al}_{2} \mathrm{O}_{3}{ }^{52}$ is used as a spacer layer, an enhancement of photoluminescence strength is observed, consistent with our expectations, since none of the photogenerated carriers in $\mathrm{MoS}_{2}$ can be transferred to $\mathrm{Al}_{2} \mathrm{O}_{3}$ (Figure 3b). We anticipate that the enhanced absorption within monolayer $\mathrm{MoS}_{2}$ and charge separation at the $\mathrm{MoS}_{2} / \mathrm{NiO}_{\mathrm{x}}$ interface can be exploited for improved optoelectronic, photovoltaic and photocatalytic devices that require the unique properties of single-layer $\mathrm{MoS}_{2}$. It should be noted that placing $\mathrm{MoS}_{2}$ directly on an $\mathrm{Al}$ reflector ${ }^{53}$ instead of using an optical cavity with a dielectric spacer layer $\mathrm{MoS}_{2} / \mathrm{NiO}_{\mathrm{x}} / \mathrm{Al}$ results in a significantly reduced absorption within $\mathrm{MoS}_{2}$ even lower than on a sapphire substrate (Supporting Information S6).

To increase the absorption further, and over a broad range extending from 400 to $700 \mathrm{~nm}$ relevant for solar energy conversion devices, as opposed to narrowband spectral regions near the band gap 54 , we also fabricated and investigated plasmonic photoelectrode architectures $\mathrm{MoS}_{2} / \mathrm{Ag}$ $\mathrm{NPs} / \mathrm{NiO}_{\mathrm{x}} / \mathrm{Al}$. Our three-dimensional full-field electromagnetic simulations predict that plasmonically active Ag nanoparticles can further improve absorption within the monolayer of $\mathrm{MoS}_{2}$ to a final value of $37 \%$ for $\mathrm{Ag} \mathrm{NPs} / 45 \mathrm{~nm} \mathrm{Al}_{2} \mathrm{O}_{3} / \mathrm{Al}$ (Figure 4a, b, and Supporting Information Figure S7) and $35 \%$ for $\mathrm{Ag} \mathrm{NPs} / 40 \mathrm{~nm} \mathrm{NiO}_{\mathrm{x}} / \mathrm{Al}$ in the wavelength region from 400 to $700 \mathrm{~nm}$. We 
find that adding silver nanoparticles below $\mathrm{MoS}_{2}$ can offer the largest enhancements in light absorption as compared to Ag nanoparticles on top of $\mathrm{MoS}_{2}$ or without plasmonic Ag particles (Supporting Information S7).

Hence, in all our experimental plasmonic structures, plasmonic Ag nanoparticles were incorporated underneath the monolayer $\mathrm{MoS}_{2}$ (Figures 1 and 4). We achieved the largest broadband absorption enhancement when the plasmon resonance of the Ag NPs is located close to $670 \mathrm{~nm}$, near the band gap of $\mathrm{MoS}_{2}$. To predict the desired thickness of plasmonic Ag nanoislands embedded in air, we performed 3D FDTD simulations, and found that a thickness of 5 to $15 \mathrm{~nm}$ is optimum for maximum energy transfer to $\mathrm{MoS}_{2}$ (Supporting Information S8). The FDTD simulations were performed on structures with Ag nanodisks.

Experimentally we found that e-beam deposition of a thicker silver layer formed a thin film rather than Ag nanoislands. Hence, we deposited 5 and $7 \mathrm{~nm}$ thick Ag nanoparticles by e-beam evaporation, which resulted in particle diameters of around $30 \mathrm{~nm}$ and $100 \mathrm{~nm}$, respectively. We calculated the total absorbed fraction (Figure 1) and the absorbed fraction within different layers and within the Ag nanoparticles (Figure 4) of the structure from the E-field distributions predicted by FDTD simulations ${ }^{28,55}$ (Supporting Information S9). Figure 4a shows the simulated absorbed fraction within monolayer $\mathrm{MoS}_{2}$ for the $\mathrm{MoS}_{2} / \mathrm{Al}_{2} \mathrm{O}_{3} / \mathrm{Al}$ architecture with and without the $\mathrm{Ag}$ nanodisks (30 nm diameter, $5 \mathrm{~nm}$ thick) placed on top of the spacer layer, and for monolayer $\mathrm{MoS}_{2}$ on top of $\mathrm{Al}_{2} \mathrm{O}_{3}$ for comparison. The results with a $\mathrm{NiO}_{\mathrm{x}}$ spacer layer are essentially the same due to the nearly identical refractive index. In Figure $4 b$, the simulated absorbed fraction integrated from 400 to $700 \mathrm{~nm}$ in each layer of the total structure is shown for the photoelectrode architecture with and without Ag nanoparticles. As can be seen from the bar chart the integrated absorbed fraction within monolayer $\mathrm{MoS}_{2}$ increases from $32 \%$ (a 5x enhanced absorption compared to that 
of $\mathrm{MoS}_{2} / \mathrm{Al}_{2} \mathrm{O}_{3}$ ) to $37 \%$ (a $5.9 \mathrm{x}$ enhanced absorption compared to that of $\mathrm{MoS}_{2} / \mathrm{Al}_{2} \mathrm{O}_{3}$ ) by the incorporation of Ag nanoparticles while the absorbed fraction by the spacer and reflector layers and also the reflected fraction of the incident light decrease. Less than $11 \%$ of the incident photons are lost due to absorption in the Ag NPs (Supporting Information S18, S19). These results further support our claim of enhanced light absorption within monolayer $\mathrm{MoS}_{2}$ and support the high experimentally measured absorbed fraction of larger than $80 \%$ around $450 \mathrm{~nm}$ for $\mathrm{MoS}_{2} / \mathrm{Ag}$ $\mathrm{NPs} / \mathrm{Al}_{2} \mathrm{O}_{3} / \mathrm{Al}$ and $\mathrm{MoS}_{2} / \mathrm{Ag} \mathrm{NPs} / \mathrm{NiO}_{\mathrm{x}} / \mathrm{Al}$, with the majority of light being absorbed in the monolayer (Figure 1). The measured absorption in the total plasmonic photoelectrode structure is larger than that predicted by the simulations in the wavelength region above $450 \mathrm{~nm}$, likely due to imperfections and nonuniform coalescence of CVD-grown $\mathrm{MoS}_{2}$ triangles into a monolayer, as a result exposing some of the Ag NPs: In regions not covered with the $\mathrm{MoS}_{2}$ top layer the Ag NPs are strongly absorbing (Supporting Information 18). Furthermore, the experimentally measured spectrum is broadband due to the superposition of plasmon oscillations of different sizes, shapes, densities and couplings of Ag particles (see Supporting Information S10). Further experimental imperfections may arise from the thickness uniformity of the spacer layer and the uniformity of its optical properties, and the roughness of the aluminum substrate.

In order to distinguish whether the improved absorption within $\mathrm{MoS}_{2}$ in our architectures containing Ag NPs is mainly due to optical impedance matching effects or plasmonic near-field enhancement effects we plot the electric field energy profile along the direction of normal incident light in Figure 5a. A strong standing-wave electric field can be observed, indicating significant reflection from both architectures with and without Ag NPs, and showing that there is room left to further improve optical impedance matching of the active layer $\mathrm{MoS}_{2}$ to the incident medium. Comparing the intensity amplitudes of the near-fields in the presence of Ag NPs to those of the 
standing wave pattern at the position of the $\mathrm{MoS}_{2}$ monolayer, we find that at long wavelengths the absorption enhancement within the $\mathrm{MoS}_{2}$ monolayer can be attributed mainly to plasmonic nearfield concentration effects rather than optical impedance matching (Figure 4, Figure 5) (Note, the plasmon resonance in the absence of $\mathrm{MoS}_{2}$ is located around $662 \mathrm{~nm}$ for an $\mathrm{Al}_{2} \mathrm{O}_{3}$ and around 683 $\mathrm{nm}$ for a $\mathrm{NiO}_{\mathrm{x}}$ substrate). Figure $5 \mathrm{~b}$ shows the modulus squared of the light's electric field, and we see most enhancement in the electric field at wavelengths close to the plasmon resonance, which is also near the band gap of $\mathrm{MoS}_{2}$. A slight suppression is instead observed at $430 \mathrm{~nm}$.

Three-dimensional electromagnetic simulations can be time consuming. For this reason we also explored how well simple analytical models can predict the parameters for enhanced light absorption within our plasmonic photoelectrode structures. To gain an intuition for the optical effects of these metal nanoparticles, one may think of the layer of particles and their host medium as an effective medium. Plasmonic metals possess a real part of the refractive index $\eta$ that is much smaller than their imaginary part $\kappa$ whereas for semiconductors and dielectrics $\eta \gg \kappa$. Therefore, a composite fabricated from suitable components can be tuned to produce an effective medium fulfilling the critical coupling condition ${ }^{55,56}$ leading to perfect light absorption. To maximize the absorption rate in monolayer $\mathrm{MoS}_{2}$ we combine it with plasmonic silver nanoparticles that are known to provide the lowest optical damping in the visible region ${ }^{56}$, resulting in large E-field enhancements. Furthermore, a wide range of low-cost techniques for fabricating silver nanostructures have been developed including electron beam evaporation ${ }^{57}$, colloidal selfassembly ${ }^{58}$, nanosphere lithography ${ }^{59}$ and block copolymer lithography ${ }^{60}$. Among these techniques, ultrathin films $(5-10 \mathrm{~nm})$ of silver evaporated by e-beam physical vapor deposition present a simple one-step and reproducible way to produce nano-islands that exhibit strong local electric field enhancements. 
We tested effective medium theories for our plasmonic metamaterial consisting of three components, monolayer $\mathrm{MoS}_{2}$, and Ag nanodisks (30 nm diameter by $5 \mathrm{~nm}$ thick) embedded in air to find the most suitable one for matching our three-dimensional electromagnetic simulation results. This configuration closely resembles our experimentally realized structure. First we employed the Bruggeman effective medium approximation in two dimensions ${ }^{61}$ to find the effective dielectric constant for a two-component system of Ag nanoparticles and air. In our case, this material behaves semiconductor-like (Supporting Information S11 B). Then we used the generalized effective medium approach to combine this Ag/air composite with the $\mathrm{MoS}_{2}$ monolayer. We also tested the Maxwell-Garnett mixing rule, but found the Bruggeman model to yield much better agreement with the electromagnetic simulations (Figure 6). The integrated absorption from 400 to $700 \mathrm{~nm}$ in the total plasmonic photoelectrode structure is maximized for a Ag NPs fill fraction of 0.36 for both, the Bruggeman analytical model and numerical FDTD results. Since the FDTD simulations contain the precise information on the size and shape of the nanoparticles in contrast to the effective medium approximations (EMA), and therefore take into account the local plasmonic field enhancements there is still a finite discrepancy between FDTD and EMA results for the geometries containing nanoparticles.

In past work ${ }^{55,56}$ an optical analogue of a Salisbury screen, i.e. a structure containing a reflector layer combined with a dielectric spacer layer at the proper distance from an ultrathin absorber layer made from a plasmonic metamaterial, has been employed to achieve near-100\% absorption if the active layer fulfills the critical coupling condition:

$$
n_{e f f}+i \kappa_{e f f} \approx \frac{(1+i)\left(n_{i}-\overline{n_{s}}\right)^{\frac{1}{2}}}{\sqrt{2}}\left(\frac{\lambda_{0}}{2 \pi d_{e f f}}\right)^{\frac{1}{2}}, \text { where } \overline{n_{s}}(\lambda)=i n_{s} \cot \left(\frac{4 n_{s} h \pi}{2 \lambda}\right)
$$


Here $n_{i}$ is the refractive index for the incident medium, $n_{s}$ the refractive index for the underlying spacer layer, $d_{e f f}$ the thickness of the thin active material, $h$ is the spacer layer thickness, and $\lambda_{0}$ is the vacuum wavelength. To predict the theoretical maximum absorption in the total structure we calculated the real and imaginary refractive index for an effective medium layer that fulfills the critical coupling condition (red dash-dotted line, Figure 7) ${ }^{55,56}$. For our chosen 3-component materials system, i.e., monolayer $\mathrm{MoS}_{2}, \mathrm{Ag}$ NPs/air, it is only possible to fulfill the critical coupling condition ${ }^{55,56}$ around $450 \mathrm{~nm}$ but not over the full range from 400 to $700 \mathrm{~nm}$.

We plotted the mismatch (Supporting Information S13, S14) of the real and imaginary part of the refractive index of the effective medium layer as a function of wavelength and dielectric spacer layer thickness between the Bruggeman analytical model of our 3-component metamaterial and the effective medium model fulfilling the critical coupling condition ${ }^{55}$ (Supporting Information S12). We used the Ag NPs fill fraction of 0.36 predicted by our Bruggeman effective medium analytical model and in our transfer matrix formalism we used the complex refractive index for the aluminum back reflector when sweeping the dielectric spacer layer thickness.

We find that our 3-component metamaterial can match the real part $n_{\text {eff }}$ of the critical coupling condition at around $450 \mathrm{~nm}$ but the imaginary part $\kappa_{e f f}$ is off (Supporting Information S13, S14). Note that for this mismatch calculation the spacer layer thickness is too thin by approximately the skin depth since the analytical model for the critical coupling condition is based on the assumption of a perfect electric conductor reflector (Supporting Information S12-S14). We conclude that our Bruggeman effective medium model can predict the optimum Ag fill fraction and dielectric spacer layer thickness in very good agreement with the numerical simulations, and it predicts that the mismatch of the real part $n_{e f f}$ w.r.t. the critical coupling condition is zero around $450 \mathrm{~nm}$ but the 
imaginary part $\kappa_{e f f}$ is off at all wavelengths. It predicts a similar total absorbed fraction in our plasmonic photoelectrode architecture as the numerical simulations (Figure 7).

The detailed analyses and tools developed here will serve as a platform for the design of nextgeneration photonic, optoelectronic and photocatalytic devices created from 2D materials. Specifically, we demonstrated a simple plasmonic architecture $\mathrm{MoS}_{2} / \mathrm{AgNPs} / \mathrm{NiO}_{\mathrm{x}} / \mathrm{Al}$ suitable as a photoelectrode to drive photochemical reactions that is numerically predicted to achieve broadband light absorption of $35 \%$ within a single layer of $\mathrm{MoS}_{2}$, while simultaneously facilitating charge separation. This absorption is on par with results of a recent numerical study that demonstrated broadband absorption up to $33 \%$ in a more complicated chirped-planar-dielectric cavity architecture ${ }^{24}$ for photodetector applications. Our approach can be easily extended to achieve improved absorption in other photoelectrodes based on two-dimensional metal dichalcogenides. With these results, we have taken a first step towards designing a photoelectrode architecture that utilizes the unique electronic and catalytic ${ }^{11,16}$ properties of monolayer $\mathrm{MoS}_{2}-\mathrm{a}$ known nonprecious and efficient hydrogen evolution catalyst ${ }^{11}$, while employing photon management strategies to turn monolayer $\mathrm{MoS}_{2}$ into a more efficient light absorber. For semiconducting $\mathrm{MoS}_{2}$, it is commonly assumed that the hydrogen evolution reaction in solar water splitting occurs at the edge sites. Therefore, monolayer-based $\mathrm{MoS}_{2}$ photoelectrodes may benefit from the introduction of discontinuities, gaps or lateral nanostructures to expose the edge sites efficiently. The effects of the inclusion of Ag nanoparticles, including size- and shape-dependent effects, on the optical, electronic and catalytic properties are beyond the scope of this work. As is commonly done in the current literature, we made the assumption that the dielectric constant of $\mathrm{MoS}_{2}$ is isotropic in this highly anisotropic material. However, the expected anisotropy of the optical properties of monolayer $\mathrm{MoS}_{2}$ warrants further studies. The models and tools developed 
here may serve as a starting point for future studies of optical metamaterials ${ }^{62}$ created from $2 \mathrm{D}$ materials.

\section{Methods}

We fabricated our structures by first depositing a $200 \mathrm{~nm}$ thick aluminum film via electron beam evaporation on a p-type silicon substrate. Next, we deposited either a $\sim 40 \mathrm{~nm}$ thick $\mathrm{NiO}_{\mathrm{x}}$ film by spin-coating, or $40 \mathrm{~nm}$ and $45 \mathrm{~nm}$ thick $\mathrm{Al}_{2} \mathrm{O}_{3}$ films by e-beam evaporation as the wide band gap dielectric spacer layer. We prepared $\mathrm{NiO}_{x}$ via a two-step sol-gel method that we adapted following a previously reported recipe ${ }^{50,63}$. We measured the complex refractive index of both dielectric spacer layers (Supporting Information S15). For our second architecture employing plasmonic silver nanoparticles $\mathrm{MoS}_{2} / \mathrm{Ag} \mathrm{NPs} / \mathrm{NiO}_{\mathrm{x}} / \mathrm{Al}$, we deposited $5 \mathrm{~nm}$ thick $\mathrm{Ag}$ nanoislands as determined by a quartz crystal oscillator thickness monitor. SEM images (Supporting Information S10) show elongated Ag hemispheres, with diameters varying from 10 to $50 \mathrm{~nm}$ and an average inter-particle distance of less than $5 \mathrm{~nm}$ at a surface concentration of $<50 \%$. The transfer process of $\mathrm{MoS}_{2}$ onto the $\mathrm{Ag} \mathrm{NP/Spacer/Al} \mathrm{substrate} \mathrm{is} \mathrm{described} \mathrm{in} \mathrm{the} \mathrm{Supporting} \mathrm{Information} \mathrm{S23.}$

Monolayer $\mathrm{MoS}_{2}$ films were grown by chemical vapor deposition (CVD) by exposing $\mathrm{MoO}_{3}$ powder to sulfur vapor at $700{ }^{\circ} \mathrm{C}$ temperature using argon as a carrier gas ${ }^{64-66}$. We synthesized millimeter scale monolayer $\mathrm{MoS}_{2}$ (Supporting Information S16). To verify regions of monolayer growth we utilized Raman spectroscopy and ensured the two Raman active modes in a $\mathrm{MoS}_{2}$ exhibit the characteristic wavenumber difference of fewer than $21 \mathrm{~cm}^{-1}$ between the in-plane and the out-of-plane modes ${ }^{67}$. We also determined a thickness of $0.8 \mathrm{~nm}$ by atomic force microscopy (AFM) measurements ${ }^{67}$ further supporting monolayer growth. We determined the complex dielectric function of monolayer $\mathrm{MoS}_{2}$ by measuring the reflectance and transmittance as a function of wavelength ${ }^{68}$. Photoluminescence and Raman measurements were carried out on a 
confocal Raman microscope. To avoid local heating and thermal etching in this experiment ${ }^{69}$, we followed a careful alignment with a laser power of $1.25 \mathrm{~mW}^{70}$.

Our optical characterization measurements were performed with an integrating sphere that takes into account both specular reflection and diffuse scattering. Hence, our inferred absorption values are accurate even in the presence of rough, scattering samples.

'Supporting Information Available: Reflection microscope images of $\mathrm{MoS}_{2}$ on different substrates, thin film SEM characterization of the plasmonic nanoparticles, Raman spectroscopy and photoluminescence of $\mathrm{MoS}_{2}$ on different substrates, optical characterization of $\mathrm{Al}_{2} \mathrm{O}_{3}$ and $\mathrm{NiO}_{x}$, electromagnetic simulations/ absorbed fraction in monolayer $\mathrm{MoS}_{2}$ and in total photoelectrode with and without plasmonic nanoparticles of varying material, size, size distributions, shape and location with respect to monolayer $\mathrm{MoS}_{2}$, results and comparisons of simulations and analytical models, discussion of possible mechanisms of photoluminescence quenching. This material is available free of charge via the Internet at http://pubs.acs.org/.'

ACKNOWLEDGMENT We would like to thank group members in Dr. Pulickel M Ajayan's group whose advice helped us to understand the chemistry of monolayer $\mathrm{MoS}_{2}$. We especially acknowledge Elizabeth F Bianco for her collaboration during the synthesis of monolayer $\mathrm{MoS}_{2}$ and Dr. Antony George for his valuable suggestions on exfoliation of $\mathrm{MoS}_{2}$. We gratefully acknowledge support from the Robert A. Welch Foundation (Grant No. C-1825) and the National Science Foundation (NSF CAREER Award, CHE- 1352579, and the NSF MRI CHE-1428184).

\section{Notes}

The authors declare no competing financial interest. 

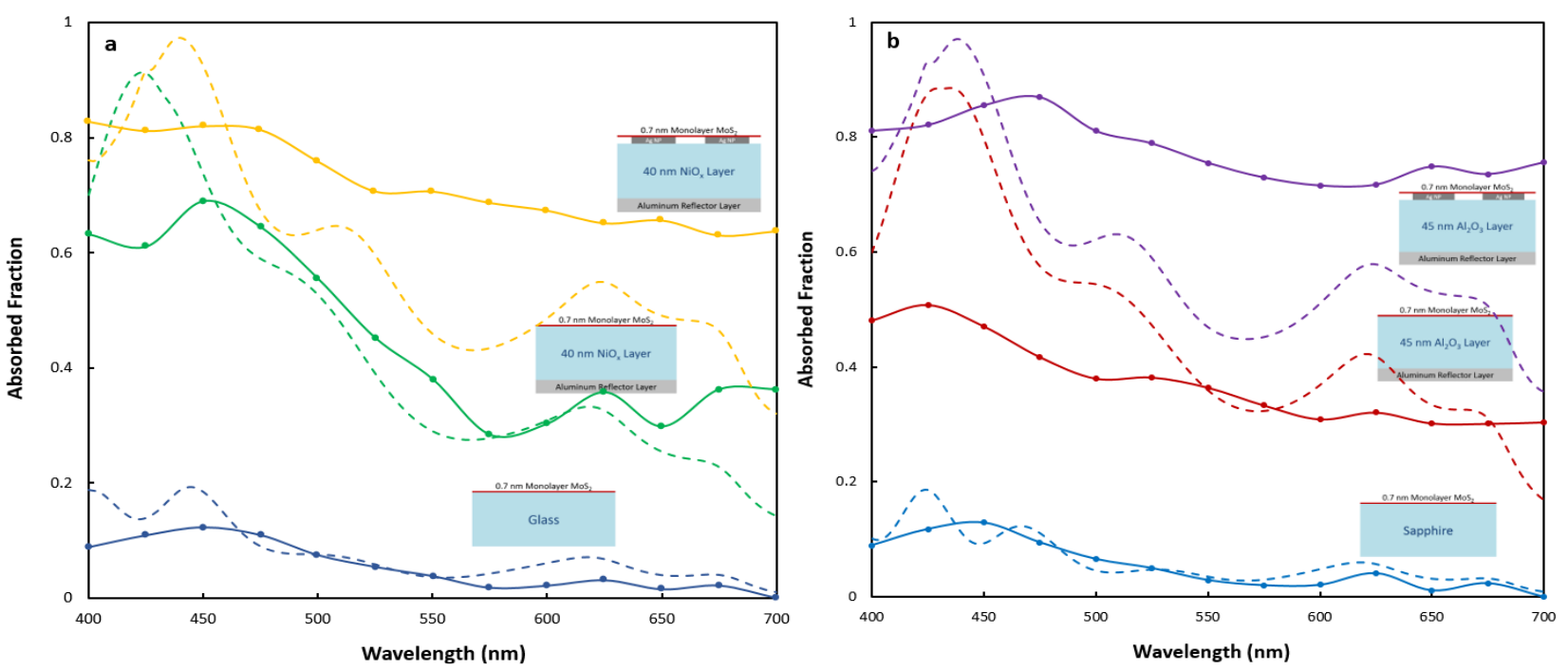

Figure 1. Absorption engineering in planar $\mathrm{MoS}_{2}$ architectures: Experimental results (solid lines) and electromagnetic simulations (dashed lines) used to predict structural parameters. (a) (i) Blue: $\mathrm{MoS}_{2} / \mathrm{SiO}_{2}$ as benchmark. (ii) Green: To achieve better light absorption within monolayer $\mathrm{MoS}_{2}$ we explore improved optical impedance matching in a geometry consisting of $\mathrm{MoS}_{2}$ on top of a wide band gap dielectric spacer $\mathrm{NiO}_{\mathrm{x}}$ and an $\mathrm{Al}$ reflector, i.e. $\mathrm{MoS}_{2} / 40 \mathrm{~nm} \mathrm{NiO}$ / $\mathrm{Al}$. Furthermore, $\mathrm{NiO}_{\mathrm{x}}$ is chosen as a spacer layer because it can serve as a scavenger of the photoholes generated in $\mathrm{MoS}_{2}$ in addition to providing suitable optical properties for optical impedance matching. (iii) Yellow: Plasmonic silver nanoparticles can further enhance the absorption within monolayer $\mathrm{MoS}_{2}$ in a $\mathrm{MoS}_{2}$ monolayer/ Ag nanoparticles $/ \mathrm{NiO}_{\mathrm{x}}$ dielectric spacer / $\mathrm{Al}$ reflector geometry. (b) Same as in Figure 1a, but with an $\mathrm{Al}_{2} \mathrm{O}_{3}$ dielectric spacer layer, i.e. $\mathrm{MoS}_{2} / 45 \mathrm{~nm} \mathrm{Al} \mathrm{O}_{3} / \mathrm{Al}$, and substrate as a benchmark, i.e. $\mathrm{MoS}_{2} / \mathrm{Al}_{2} \mathrm{O}_{3}$. Note that the absorption spectra calculated by FDTD exhibit slight oscillatory artifacts arising from a polynomial fit to the real and imaginary part of the dielectric constant that causes an overestimation of the absorption around $440 \mathrm{~nm}$. 


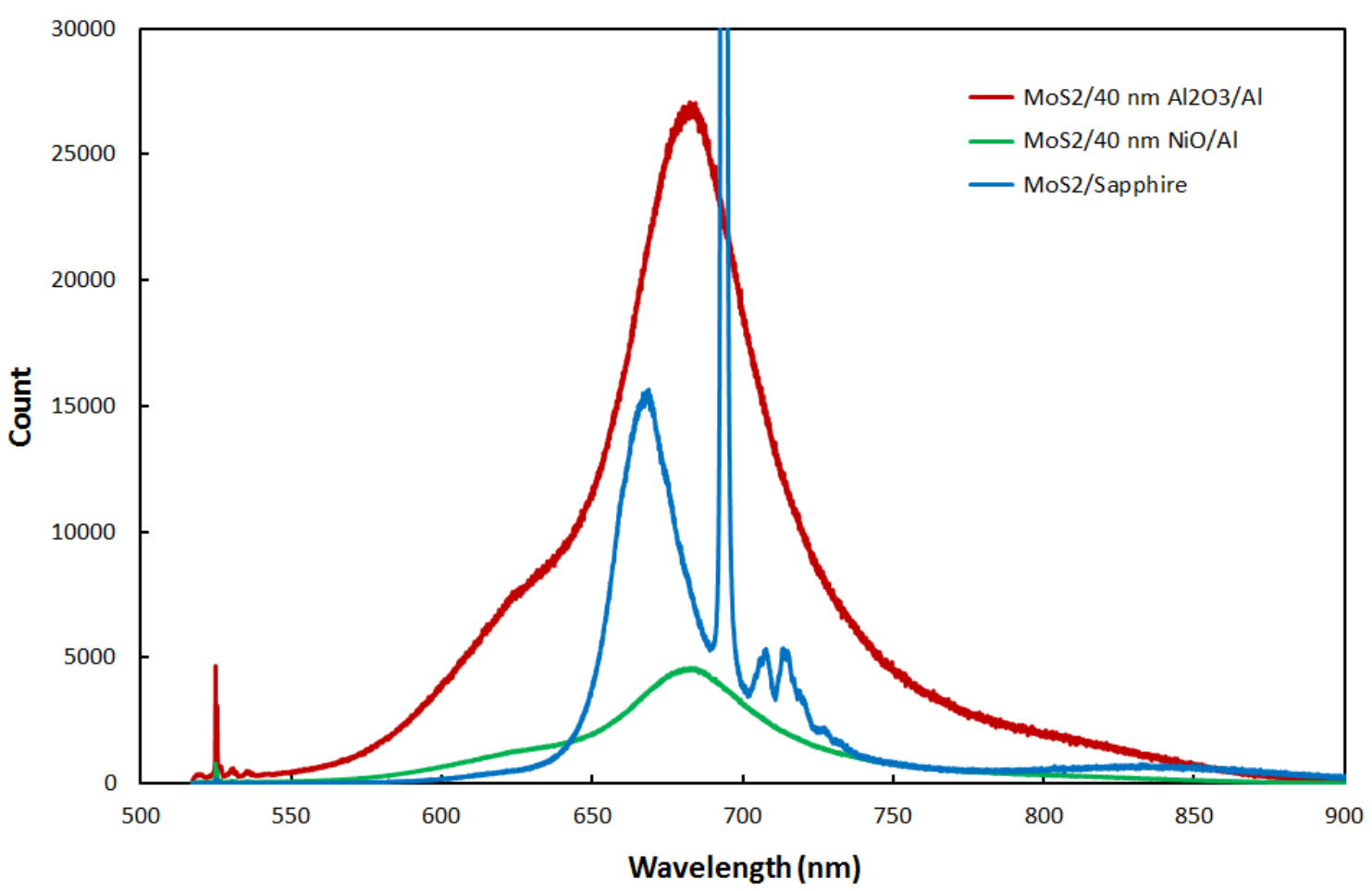

Figure 2. Photoluminescence spectra, excited at $514.5 \mathrm{~nm}$, for (i) $\mathrm{MoS}_{2}$ on $\mathrm{Al}_{2} \mathrm{O}_{3}$ (blue), (ii) $\mathrm{MoS}_{2}$ on $40 \mathrm{~nm} \mathrm{Al} \mathrm{O}_{3} / \mathrm{Al}$ (red), and (iii) our target structure $\mathrm{MoS}_{2}$ on $\mathrm{NiO}_{\mathrm{x}} / \mathrm{Al}$ designed for efficient charge carrier separation (green). The photoluminescence intensity for planar monolayer $\mathrm{MoS}_{2}$ on $40 \mathrm{~nm} \mathrm{Al} \mathrm{A}_{2} \mathrm{O}_{3}$ with an $\mathrm{Al}$ reflector (structure (ii)) is increased three times compared to planar monolayer $\mathrm{MoS}_{2}$ on $\mathrm{Al}_{2} \mathrm{O}_{3}$ (structure (i)), which supports enhanced absorption in the better optically impedance matched structure. In contrast, in $\mathrm{MoS}_{2}$ on $\mathrm{NiO}_{\mathrm{x}} / \mathrm{Al}$ (structure (iii)) the photoluminescence spectrum shows luminescence quenching compared to (i), which we attribute to hole scavenging by the underlying $\mathrm{NiO}_{\mathrm{x}}$ substrate (green). Note that the difference in luminescence between (ii) and (iii) is unlikely to be due to a difference in absorption, since the refractive index for both wide band gap dielectric spacer layers $\mathrm{NiO}_{\mathrm{x}}$ and $\mathrm{Al}_{2} \mathrm{O}_{3}$ is approximately $\mathrm{n}=1.8$, and since simulations predict similar absorption over the full wavelength range. 
a)

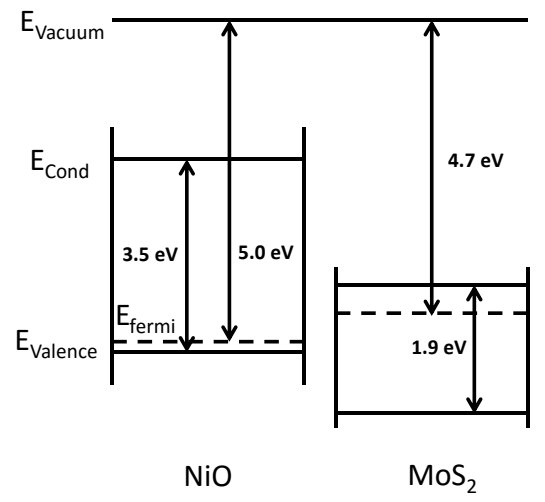

b)

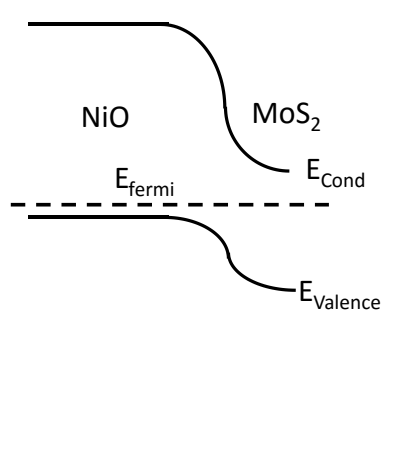

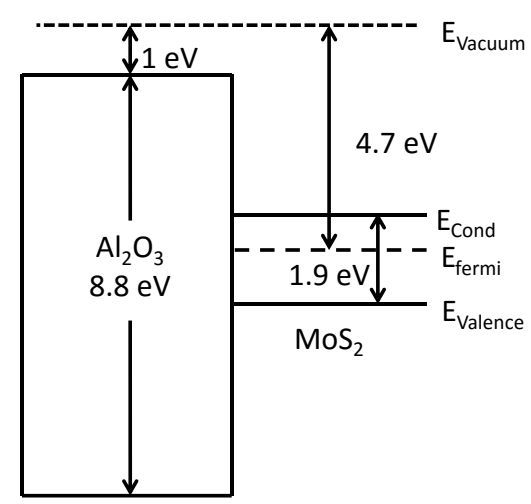

Figure 3. Energy schematic of our structure (a) Left: The energy band diagram for $\mathrm{MoS}_{2}$ and $\mathrm{NiO}_{x}$ shows the relative positions of the Fermi levels with respect to the vacuum level before establishing a $\mathrm{MoS}_{2}-\mathrm{NiO}_{\mathrm{x}}$ contact. Right: The energy band diagram of $\mathrm{MoS}_{2}-\mathrm{NiO}_{\mathrm{x}}$ showing band bending after establishing the contact between $\mathrm{NiO}_{\mathrm{x}}$ and $\mathrm{MoS}_{2} . \mathrm{MoS}_{2}$ on $\mathrm{NiO}_{\mathrm{x}} / \mathrm{Al}$ does not show significant photoluminescence consistent with the presence of an efficient charge separation mechanism in $\mathrm{MoS}_{2}$ on $\mathrm{NiO}_{\mathrm{x}} / \mathrm{Al}$. (b) Band diagram of $\mathrm{MoS}_{2}$ on top of $\mathrm{Al}_{2} \mathrm{O}_{3} /$ Aluminum. Because of the large energy barrier of $\mathrm{Al}_{2} \mathrm{O}_{3}$, photogenerated charge carriers cannot be transferred from $\mathrm{MoS}_{2}$ to $\mathrm{Al}_{2} \mathrm{O}_{3}$, consistent with the observed highly efficient photoluminescence from this structure. 

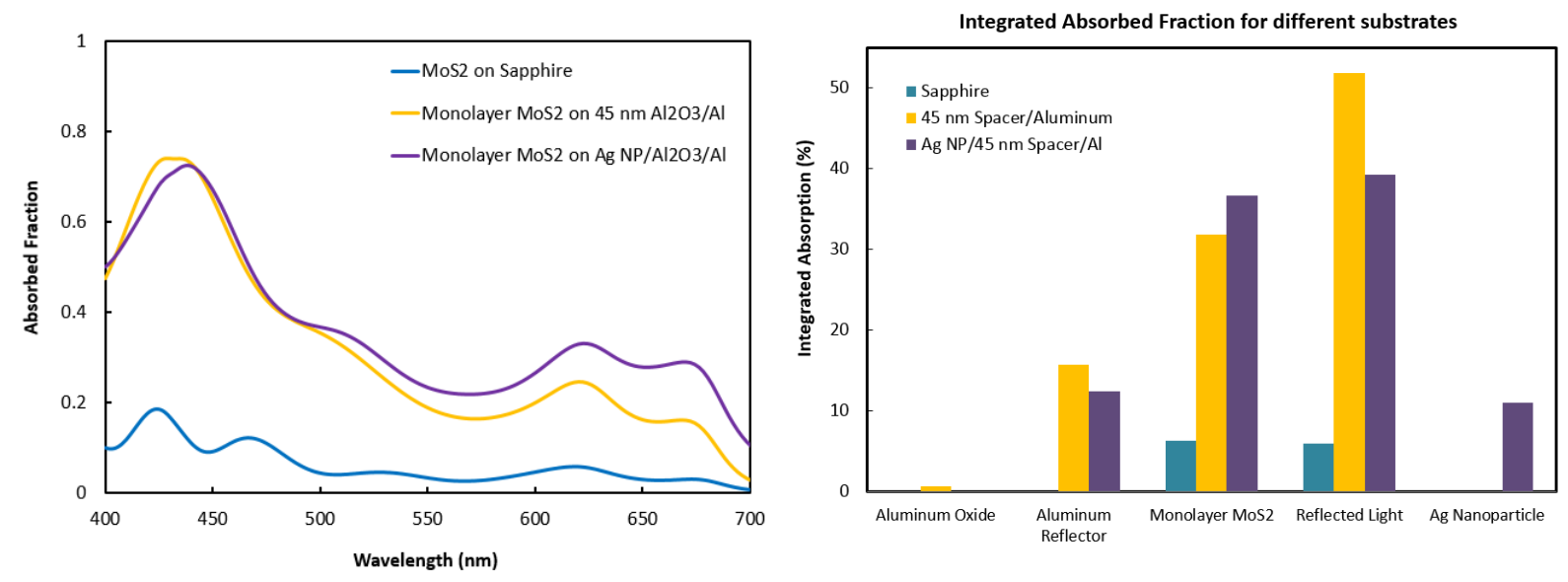

Figure 4. Simulated fraction of light absorbed within a monolayer of $\mathrm{MoS}_{2}$, as a function of wavelength. (i) on $\mathrm{Al}_{2} \mathrm{O}_{3}$ (blue line), (ii) on $45 \mathrm{~nm} \mathrm{Al}_{2} \mathrm{O}_{3} / \mathrm{Al}$ (yellow line), and (iii) on $\mathrm{Ag}$ nanoparticles/45 $\mathrm{nm} \mathrm{Al} \mathrm{Al}_{2} / \mathrm{Al}$ (purple line). These simulation results predict absorption enhancements within the $7 \AA$ thick monolayer $\mathrm{MoS}_{2}$ when placed on substrates (ii) and (iii). (b) Comparison of the absorbed fraction (integrated from $400 \mathrm{~nm}$ to $700 \mathrm{~nm}$ ) by individual components in a structure with Ag nanoparticles (purple) and without Ag nanoparticles (yellow). The fraction of light reflected is shown for comparison. The integrated absorbed fraction within monolayer $\mathrm{MoS}_{2}$ increases by $16 \%$ when $\mathrm{Ag}$ nanoparticles are incorporated, whereas the absorbed fraction by the spacer and reflector layer and the reflected fraction of light decrease. 

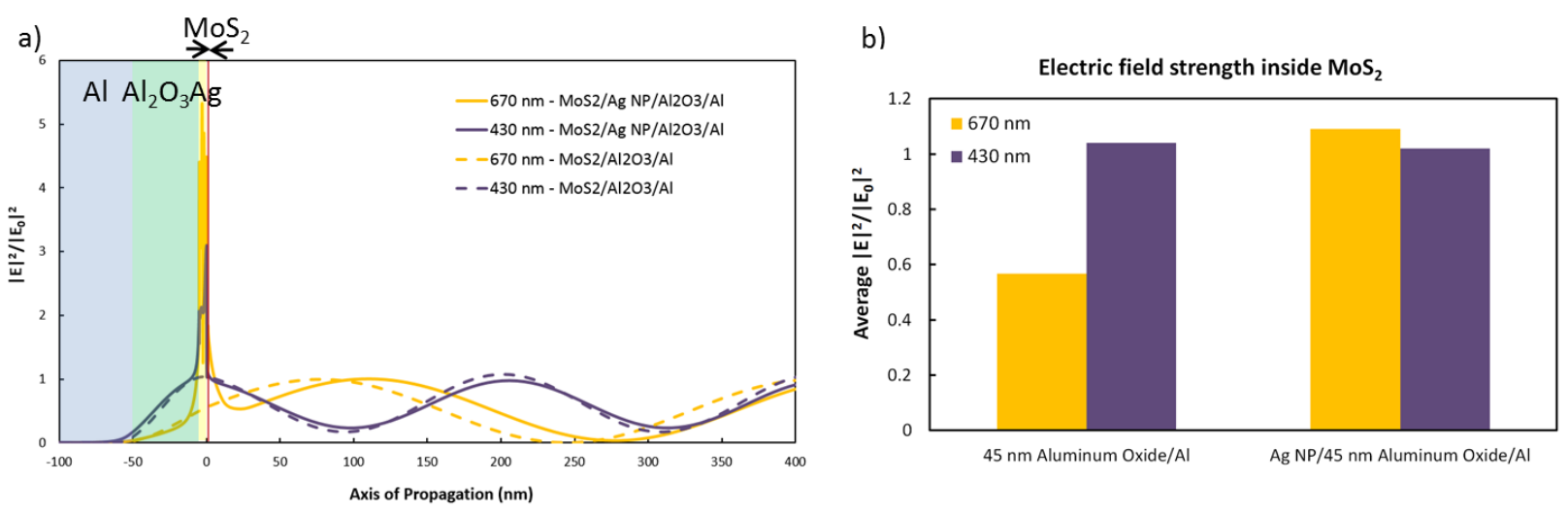

Figure 5. Optical impedance matching vs. plasmonic near-field enhancement effects for improved absorption within $\mathrm{MoS}_{2}$. (a) Left: Modulus squared of the light's electric field along its axis of propagation for a structure without $\left(\mathrm{MoS}_{2} / \mathrm{Al}_{2} \mathrm{O}_{3} / \mathrm{Al}\right)$ and with $\mathrm{Ag}$ nanoparticles $\left(\mathrm{MoS}_{2} / \mathrm{AgNPs} /\right.$ $\mathrm{Al}_{2} \mathrm{O}_{3} / \mathrm{Al}$ ), plotted near the wavelength of peak absorption of $\mathrm{MoS}_{2}$ at $430 \mathrm{~nm}$ (purple line) and near the bandgap of $\mathrm{MoS}_{2}$ at $670 \mathrm{~nm}$ (yellow line). Each component of the structure is shown in a different color: Blue as reflector layer, green as spacer layer, yellow as Ag nanoparticle layer and red as $\mathrm{MoS}_{2}$ absorber layer. (b) The bar chart shows a comparison of the modulus squared of the electric field within monolayer $\mathrm{MoS}_{2}$ for the two structures with and without Ag NPs at $430 \mathrm{~nm}$ and $670 \mathrm{~nm}$. In our structure, the absorption enhancement in $\mathrm{MoS}_{2}$ in the presence of $\mathrm{Ag}$ nanoparticles originates predominantly from near-field concentration effects brought about by the Ag nanoparticles. 

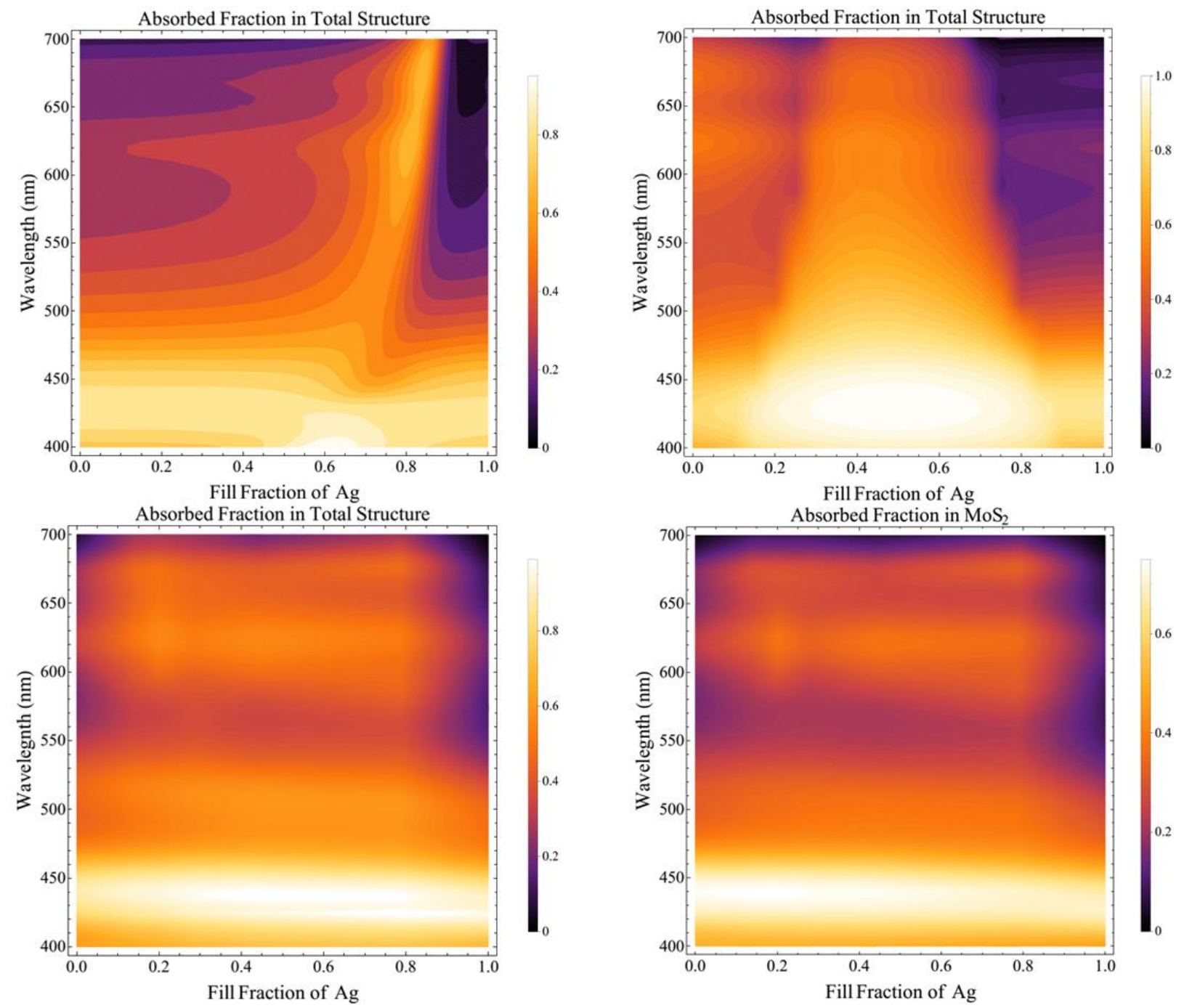

Figure 6. Top left: Absorbed fraction of the incident light in the total plasmonic photoelectrode structure $\mathrm{MoS}_{2} / \mathrm{Ag} \mathrm{NPs} / 45 \mathrm{~nm} \mathrm{Al} \mathrm{O}_{3} / \mathrm{Al}$ as a function of wavelength and $\mathrm{Ag}$ nanoparticle fill fraction determined by a transfer matrix model employing the Maxwell Garnett effective medium approximation. Top Right: Absorbed fraction of the incident light in the total plasmonic photoelectrode structure $\mathrm{MoS}_{2} / \mathrm{Ag} \mathrm{NPs} / 45 \mathrm{~nm} \mathrm{Al} \mathrm{O}_{3} / \mathrm{Al}$ as a function of wavelength and $\mathrm{Ag}$ nanoparticle fill fraction determined by a transfer matrix model employing the Bruggeman effective medium approximation. Bottom Left: Absorbed fraction of the incident light in the total plasmonic photoelectrode structure $\mathrm{MoS}_{2} / \mathrm{Ag} \mathrm{NPs} / 45 \mathrm{~nm} \mathrm{Al}{ }_{2} \mathrm{O}_{3} / \mathrm{Al}$ as a function of wavelength and Ag nanoparticle fill fraction determined by FDTD simulations. Bottom Right: Absorbed 
fraction of the incident light only within the monolayer of $\mathrm{MoS}_{2}$ for the plasmonic photoelectrode

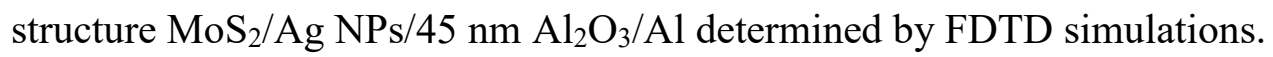

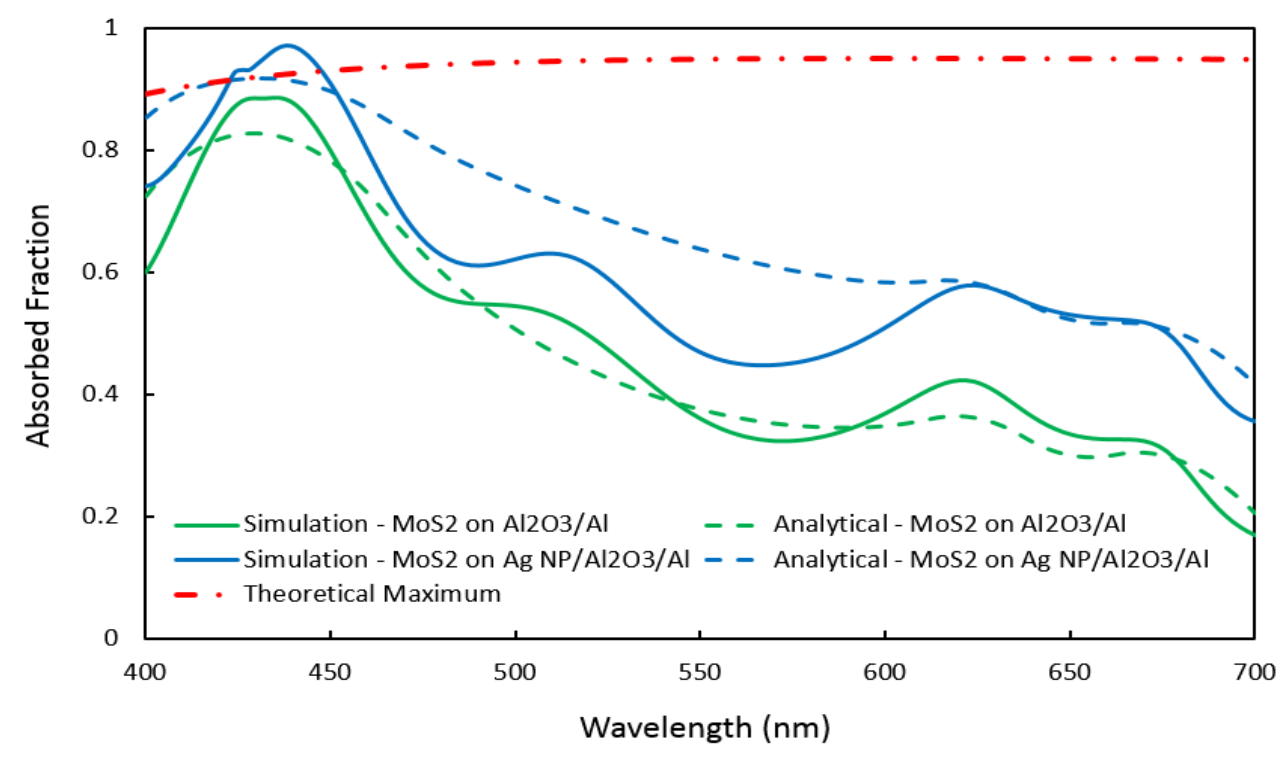

Figure 7. Comparison of the absorption by the total structure predicted by numerical simulations and the analytical model based on the Bruggeman effective medium approximation. The results are also compared with respect to the theoretical maximum absorption that can be achieved if one can match the refractive indices predicted by equation (1), i.e. the analytical model for the critical coupling condition. 


\section{REFERENCES}

(1) Conley, H. J.; Wang, B.; Ziegler, J. I.; Haglund, R. F.; Pantelides, S. T.; Bolotin, K. I. Bandgap Engineering of Strained Monolayer and Bilayer MoS2. Nano Lett. 2013, 13, 36263630 .

(2) Newaz, A. K. M.; Prasai, D.; Ziegler, J. I.; Caudel, D.; Robinson, S.; Haglund, R. F.; Bolotin, K. I. Electrical control of optical properties of monolayer MoS2. Solid State Commun. 2013, 155, 49-52.

(3) Voiry, D.; Goswami, A.; Kappera, R.; SilvaCecilia de Carvalho Castro, e.; Kaplan, D.; Fujita, T.; Chen, M.; Asefa, T.; Chhowalla, M. Covalent functionalization of monolayered transition metal dichalcogenides by phase engineering. Nat. Chem. 2015, 7, 45-49.

(4) Yu, W. J.; Liu, Y.; Zhou, H.; Yin, A.; Li, Z.; Huang, Y.; Duan, X. Highly efficient gate-tunable photocurrent generation in vertical heterostructures of layered materials. Nat. Nanotechnol. 2013, 8, 952-958.

(5) Pospischil, A.; Furchi, M. M.; Mueller, T. Solar-energy conversion and light emission in an atomic monolayer p-n diode. Nat. Nanotechnol. 2014, 9, 257-261.

(6) Jariwala, D.; Sangwan, V. K.; Lauhon, L. J.; Marks, T. J.; Hersam, M. C. Emerging device applications for semiconducting two-dimensional transition metal dichalcogenides. ACS Nano 2014, 8, 1102-1120.

(7) Butun, S.; Tongay, S.; Aydin, K. Enhanced Light Emission from Large-Area Monolayer MoS2 Using Plasmonic Nanodisc Arrays. Nano Lett. 2015, 15, 2700-2704.

(8) Wu, C. C.; Jariwala, D.; Sangwan, V. K.; Marks, T. J.; Hersam, M. C.; Lauhon, L. J. Elucidating the Photoresponse of Ultrathin MoS2 Field-Effect Transistors by Scanning Photocurrent Microscopy. J. Phys. Chem. Lett. 2013, 4, 2508-2513.

(9) Jariwala, D.; Sangwan, V. K.; Wu, C. C.; Prabhumirashi, P. L.; Geier, M. L.; Marks, T. J.; Lauhon, L. J.; Hersam, M. C. Gate-tunable carbon nanotube-MoS2 heterojunction p-n diode. Proc. Natl. Acad. Sci. U. S. A. 2013, 110, 18076-18080.

(10) Jaramillo, T. F.; Jorgensen, K. P.; Bonde, J.; Nielsen, J. H.; Horch, S.; Chorkendorff, I. Identification of active edge sites for electrochemical H2 evolution from MoS2 nanocatalysts. Science 2007, 317, 100-102.

(11) Laursen, A. B.; Kegnaes, S.; Dahl, S.; Chorkendorff, I. Molybdenum sulfidesefficient and viable materials for electro - and photoelectrocatalytic hydrogen evolution. Energy Environ. Sci. 2012, 5, 5577-5591.

(12) Xiang, Q.; Yu, J. Graphene-Based Photocatalysts for Hydrogen Generation. J. Phys. Chem. Lett. 2013, 4, 753-759.

(13) Bernardi, M.; Palummo, M.; Grossman, J. C. Extraordinary sunlight absorption and one nanometer thick photovoltaics using two-dimensional monolayer materials. Nano Lett. 2013, 13, 3664-3670.

(14) Meng, F. K.; Li, J. T.; Cushing, S. K.; Zhi, M. J.; Wu, N. Q. Solar Hydrogen Generation by Nanoscale p-n Junction of p-type Molybdenum Disulfide/n-type Nitrogen-Doped Reduced Graphene Oxide. J. Am. Chem. Soc. 2013, 135, 10286-10289.

(15) Tsai, M.-L.; Su, S.-H.; Chang, J.-K.; Tsai, D.-S.; Chen, C.-H.; Wu, C.-I.; Li, L.-J.; Chen, L.-J.; He, J.-H. Monolayer MoS2 Heterojunction Solar Cells. ACS Nano 2014, 8, 83178322 .

(16) Kang, J.; Tongay, S.; Zhou, J.; Li, J. B.; Wu, J. Q. Band offsets and heterostructures of two-dimensional semiconductors. Appl. Phys. Lett. 2013, 102. 
(17) Pan, J.; Wang, Z.; Chen, Q.; Hu, J.; Wang, J. Band structure engineering of monolayer $\operatorname{MoS}(2)$ by surface ligand functionalization for enhanced photoelectrochemical hydrogen production activity. Nanoscale 2014, 6, 13565-13571.

(18) Chen, Z.; Cummins, D.; Reinecke, B. N.; Clark, E.; Sunkara, M. K.; Jaramillo, T. F. Core-shell MoO3-MoS2 nanowires for hydrogen evolution: a functional design for electrocatalytic materials. Nano Lett. 2011, 11, 4168-4175.

(19) Wang, H.; Lu, Z.; Xu, S.; Kong, D.; Cha, J. J.; Zheng, G.; Hsu, P. C.; Yan, K.; Bradshaw, D.; Prinz, F. B.; Cui, Y. Electrochemical tuning of vertically aligned MoS2 nanofilms and its application in improving hydrogen evolution reaction. Proc. Natl. Acad. Sci. U. S. A. 2013, 110, 19701-19706.

(20) Wang, H.; Zhang, Q.; Yao, H.; Liang, Z.; Lee, H. W.; Hsu, P. C.; Zheng, G.; Cui, Y. High electrochemical selectivity of edge versus terrace sites in two-dimensional layered MoS2 materials. Nano Lett. 2014, 14, 7138-7144.

(21) Li, Y.; Wang, H.; Xie, L.; Liang, Y.; Hong, G.; Dai, H. MoS2 nanoparticles grown on graphene: an advanced catalyst for the hydrogen evolution reaction. J. Am. Chem. Soc. 2011, 133, 7296-7299.

(22) Lopez-Sanchez, O.; Lembke, D.; Kayci, M.; Radenovic, A.; Kis, A. Ultrasensitive photodetectors based on monolayer MoS2. Nat. Nanotechnol. 2013, 8, 497-501.

(23) Cheng, R.; Li, D.; Zhou, H.; Wang, C.; Yin, A.; Jiang, S.; Liu, Y.; Chen, Y.; Huang, Y.; Duan, X. Electroluminescence and photocurrent generation from atomically sharp WSe2/MoS2 heterojunction p-n diodes. Nano Lett. 2014, 14, 5590-5597.

(24) Zheng, J. B.; Barton, R. A.; Englund, D. Broadband Coherent Absorption in Chirped-Planar-Dielectric Cavities for 2D-Material-Based Photovoltaics and Photodetectors. ACS Photonics 2014, 1, 768-774.

(25) Liu, J. T.; Wang, T. B.; Li, X. J.; Liu, N. H. Enhanced absorption of monolayer MoS2 with resonant back reflector. J. Appl. Phys. 2014, 115.

(26) Wang, W. Y.; Klots, A.; Yang, Y. M.; Li, W.; Kravchenko, I. I.; Briggs, D. P.; Bolotin, K. I.; Valentine, J. Enhanced absorption in two-dimensional materials via Fano-resonant photonic crystals. Appl. Phys. Lett. 2015, 106.

(27) Akselrod, G. M.; Ming, T.; Argyropoulos, C.; Hoang, T. B.; Lin, Y.; Ling, X.; Smith, D. R.; Kong, J.; Mikkelsen, M. H. Leveraging Nanocavity Harmonics for Control of Optical Processes in 2D Semiconductors. Nano Lett. 2015, 15, 3578-3584.

(28) Thomann, I.; Pinaud, B. A.; Chen, Z.; Clemens, B. M.; Jaramillo, T. F.; Brongersma, M. L. Plasmon Enhanced Solar-to-Fuel Energy Conversion. Nano Lett. 2011, 11, 3440-3446.

(29) Schweikhard, V.; Grubisic, A.; Baker, T. A.; Thomann, I.; Nesbitt, D. J. Polarization-Dependent Scanning Photoionization Microscopy: Ultrafast Plasmon-Mediated Electron Ejection Dynamics in Single Au Nanorods. ACS Nano 2011, 5, 3724-3735.

(30) Schweikhard, V.; Grubisic, A.; Baker, T. A.; Nesbitt, D. J. Multiphoton Scanning Photoionization Imaging Microscopy for Single-Particle Studies of Plasmonic Metal Nanostructures. J. Phys. Chem. C 2011, 115, 83-91.

(31) Britnell, L.; Ribeiro, R. M.; Eckmann, A.; Jalil, R.; Belle, B. D.; Mishchenko, A.; Kim, Y. J.; Gorbachev, R. V.; Georgiou, T.; Morozov, S. V.; Grigorenko, A. N.; Geim, A. K.; Casiraghi, C.; Castro Neto, A. H.; Novoselov, K. S. Strong light-matter interactions in heterostructures of atomically thin films. Science 2013, 340, 1311-1314. 
(32) Sobhani, A.; Lauchner, A.; Najmaei, S.; Ayala-Orozco, C.; Wen, F.; Lou, J.; Halas, N. J. Enhancing the photocurrent and photoluminescence of single crystal monolayer MoS2 with resonant plasmonic nanoshells. Appl. Phys. Lett. 2014, 104, 031112.

(33) Buscema, M.; Steele, G.; van der Zant, H. J.; Castellanos-Gomez, A. The effect of the substrate on the Raman and photoluminescence emission of single-layer MoS2. Nano Res. 2014, 7, 561-571.

(34) Eda, G.; Yamaguchi, H.; Voiry, D.; Fujita, T.; Chen, M.; Chhowalla, M. Photoluminescence from Chemically Exfoliated MoS2. Nano Lett. 2011, 11, 5111-5116.

(35) Sun, Y. H.; Liu, K.; Hong, X. P.; Chen, M.; Kim, J.; Shi, S. F.; Wu, J. Q.; Zettl, A.; Wang, F. Probing Local Strain at MX2-Metal Boundaries with Surface Plasmon-Enhanced Raman Scattering. Nano Lett. 2014, 14, 5329-5334.

(36) Lien, D. H.; Kang, J. S.; Amani, M.; Chen, K.; Tosun, M.; Wang, H. P.; Roy, T.; Eggleston, M. S.; Wu, M. C.; Dubey, M.; Lee, S. C.; He, J. H.; Javey, A. Engineering light outcoupling in 2D materials. Nano Lett. 2015, 15, 1356-1361.

(37) Yin, Z.; Li, H.; Li, H.; Jiang, L.; Shi, Y.; Sun, Y.; Lu, G.; Zhang, Q.; Chen, X.; Zhang, H. Single-Layer MoS2 Phototransistors. ACS Nano 2012, 6, 74-80.

(38) Liu, K.-K.; Zhang, W.; Lee, Y.-H.; Lin, Y.-C.; Chang, M.-T.; Su, C.-Y.; Chang, C.-S.; Li, H.; Shi, Y.; Zhang, H.; Lai, C.-S.; Li, L.-J. Growth of Large-Area and Highly Crystalline MoS2 Thin Layers on Insulating Substrates. Nano Lett. 2012, 12, 1538-1544.

(39) Bhanu, U.; Islam, M. R.; Tetard, L.; Khondaker, S. I. Photoluminescence quenching in gold - MoS2 hybrid nanoflakes. Sci. Rep. 2014, 4.

(40) Tian, H.; Oscarsson, J.; Gabrielsson, E.; Eriksson, S. K.; Lindblad, R.; Xu, B.; Hao, Y.; Boschloo, G.; Johansson, E. M. J.; Gardner, J. M.; Hagfeldt, A.; Rensmo, H.; Sun, L. Enhancement of p-Type Dye-Sensitized Solar Cell Performance by Supramolecular Assembly of Electron Donor and Acceptor. Sci. Rep. 2014, 4.

(41) Wang, K.-C.; Jeng, J.-Y.; Shen, P.-S.; Chang, Y.-C.; Diau, E. W.-G.; Tsai, C.-H.; Chao, T.-Y.; Hsu, H.-C.; Lin, P.-Y.; Chen, P.; Guo, T.-F.; Wen, T.-C. p-type Mesoscopic Nickel Oxide/Organometallic Perovskite Heterojunction Solar Cells. Sci. Rep. 2014, 4.

(42) Bian, Z.; Tachikawa, T.; Cui, S.-C.; Fujitsuka, M.; Majima, T. Single-molecule charge transfer dynamics in dye-sensitized p-type NiO solar cells: influences of insulating $\mathrm{A} 12 \mathrm{O} 3$ layers. Chem. Sci. 2012, 3, 370-379.

(43) Hong, X. P.; Kim, J.; Shi, S. F.; Zhang, Y.; Jin, C. H.; Sun, Y. H.; Tongay, S.; Wu, J. Q.; Zhang, Y. F.; Wang, F. Ultrafast charge transfer in atomically thin MoS2/WS2 heterostructures. Nat. Nanotechnol. 2014, 9, 682-686.

(44) Yu, Y.; Hu, S.; Su, L.; Huang, L.; Liu, Y.; Jin, Z.; Purezky, A. A.; Geohegan, D. B.; Kim, K. W.; Zhang, Y.; Cao, L. Equally efficient interlayer exciton relaxation and improved absorption in epitaxial and nonepitaxial MoS2/WS2 heterostructures. Nano Lett. 2015, 15, 486491.

(45) Ceballos, F.; Bellus, M. Z.; Chiu, H. Y.; Zhao, H. Ultrafast charge separation and indirect exciton formation in a MoS2-MoSe2 van der Waals heterostructure. ACS Nano 2014, 8, 12717-12724.

(46) Evgenij Barsoukov, J. R. M. Impedance Spectroscopy: Theory, Experiment, and Applications, 2nd Edition, WILEY.

(47) Orazem, M. E., and Bernard Tribollet. Electrochemical impedance spectroscopy. Vol. 48. . John Wiley \& Sons 2011, 48. 
(48) Cardon, F.; Gomes, W. P. On the determination of the flat-band potential of a semiconductor in contact with a metal or an electrolyte from the Mott-Schottky plot. J. Phys. D: Appl. Phys. 1978, 11, L63.

(49) Albery, W. J.; O'Shea, G. J.; Smith, A. L. Interpretation and use of Mott-Schottky plots at the semiconductor/electrolyte interface. J. Chem. Soc., Faraday Trans. 1996, 92, 40834085.

(50) Robatjazi, H.; Bahauddin, S. M.; Doiron, C.; Thomann, I. Direct Plasmon-Driven Photoelectrocatalysis. Nano Lett. 2015, 15, 6155-6161.

(51) Wu, X.; Xing, G.; Tan, S. L. J.; Webster, R. D.; Sum, T. C.; Yeow, E. K. L. Hole transfer dynamics from dye molecules to p-type NiO nanoparticles: effects of processing conditions. Phys. Chem. Chem. Phys. 2012, 14, 9511-9519.

(52) Mohsen Yazdanmehr, S. J. A., Abolghasem Nourmohammadi, Majid Ghasemzadeh and Mahmood Rezvanian. Electronic structure and bandgap of $\gamma$-A12O3 compound using mBJ exchange potential. Nanoscale Res. Lett. 2012, 7, 488.

(53) Kats, M. A.; Blanchard, R.; Genevet, P.; Capasso, F. Nanometre optical coatings based on strong interference effects in highly absorbing media. Nat. Mater. 2013, 12, 20-24.

(54) Mak, K. F.; Lee, C.; Hone, J.; Shan, J.; Heinz, T. F. Atomically thin MoS(2): a new direct-gap semiconductor. Phys. Rev. Lett. 2010, 105, 136805.

(55) Hägglund, C.; Zeltzer, G.; Ruiz, R.; Thomann, I.; Lee, H.-B.-R.; Brongersma, M. L.; Bent, S. F. Self-Assembly Based Plasmonic Arrays Tuned by Atomic Layer Deposition for Extreme Visible Light Absorption. Nano Lett. 2013, 13, 3352-3357.

(56) Hagglund, C.; Apell, S. P. Plasmonic Near-Field Absorbers for Ultrathin Solar Cells. J. Phys. Chem. Lett. 2012, 3, 1275-1285.

(57) Wei, H. Y.; Eilers, H. From silver nanoparticles to thin films: Evolution of microstructure and electrical conduction on glass substrates. J. Phys. Chem. Solids 2009, 70, $459-465$.

(58) Tao, A. R.; Ceperley, D. P.; Sinsermsuksakul, P.; Neureuther, A. R.; Yang, P. Self-Organized Silver Nanoparticles for Three-Dimensional Plasmonic Crystals. Nano Lett. 2008, 8, 4033-4038.

(59) Hulteen, J. C.; Treichel, D. A.; Smith, M. T.; Duval, M. L.; Jensen, T. R.; Van Duyne, R. P. Nanosphere Lithography: Size-Tunable Silver Nanoparticle and Surface Cluster Arrays. The Journal of Physical Chemistry B 1999, 103, 3854-3863.

(60) Vladimir, V. T.; Olga, V. D. e.; Viktor, M. R. Formation of ordered nanoparticle assemblies by block copolymer lithography methods. Russ. Chem. Rev. 2011, 80, 453.

(61) Cai, W.; Shalaev, V. M.: Optical metamaterials fundamentals and applications. pp 1 online resource (xii, 200 pages).

(62) Cha, J. J.; Koski, K. J.; Huang, K. C.; Wang, K. X.; Luo, W.; Kong, D.; Yu, Z.; Fan, S.; Brongersma, M. L.; Cui, Y. Two-dimensional chalcogenide nanoplates as tunable metamaterials via chemical intercalation. Nano Lett. 2013, 13, 5913-5918.

(63) Sun, K.; Park, N.; Sun, Z. L.; Zhou, J. G.; Wang, J.; Pang, X. L.; Shen, S. H.; Noh, S. Y.; Jing, Y.; Jin, S. H.; Yu, P. K. L.; Wang, D. L. Nickel oxide functionalized silicon for efficient photo-oxidation of water. Energy \& Environmental Science 2012, 5, 7872-7877.

(64) Liu, Z.; Amani, M.; Najmaei, S.; Xu, Q.; Zou, X.; Zhou, W.; Yu, T.; Qiu, C.; Birdwell, A. G.; Crowne, F. J.; Vajtai, R.; Yakobson, B. I.; Xia, Z.; Dubey, M.; Ajayan, P. M.; Lou, J. Strain and structure heterogeneity in MoS2 atomic layers grown by chemical vapour deposition. Nat. Commun. 2014, 5, 5246. 
(65) Najmaei, S.; Liu, Z.; Zhou, W.; Zou, X.; Shi, G.; Lei, S.; Yakobson, B. I.; Idrobo, J. C.; Ajayan, P. M.; Lou, J. Vapour phase growth and grain boundary structure of molybdenum disulphide atomic layers. Nat. Mater. 2013, 12, 754-759.

(66) Sharma, D.; Amani, M.; Motayed, A.; Shah, P. B.; Birdwell, A. G.; Najmaei, S.; Ajayan, P. M.; Lou, J.; Dubey, M.; Li, Q.; Davydov, A. V. Electrical transport and lowfrequency noise in chemical vapor deposited single-layer MoS2 devices. Nanotechnology 2014, 25,155702 .

(67) Li, H., Zhang, Q., Yap, C. C. R., Tay, B. K., Edwin, T. H. T., Olivier, A. and Baillargeat, D. . From Bulk to Monolayer MoS2: Evolution of Raman Scattering. . Adv. Funct. Mater., 2012, 22 1385-1390.

(68) Li, Y. L.; Chernikov, A.; Zhang, X.; Rigosi, A.; Hill, H. M.; van der Zande, A. M.; Chenet, D. A.; Shih, E. M.; Hone, J.; Heinz, T. F. Measurement of the optical dielectric function of monolayer transition-metal dichalcogenides: MoS2, MoSe2, WS2, and WSe2. Phys. Rev. B 2014, 90 .

(69) Wu, J., Li, H., Yin, Z., Li, H., Liu, J., Cao, X., Zhang, Q. and Zhang, H. . Layer Thinning and Etching of Mechanically Exfoliated MoS2 Nanosheets by Thermal Annealing in Air. Small 2013, 9, 3314-3319.

(70) Castellanos-Gomez, A.; Barkelid, M.; Goossens, A. M.; Calado, V. E.; van der Zant, H. S.; Steele, G. A. Laser-thinning of MoS2: on demand generation of a single-layer semiconductor. Nano Lett. 2012, 12, 3187-3192. 